\title{
ON ISOMORPHIC CLASSICAL DIFFEOMORPHISM GROUPS. I
}

\author{
AUGUSTIN BANYAGA
}

\begin{abstract}
Let $\left(M_{i}, \alpha_{i}\right), i=1,2$, be two smooth manifolds equipped with symplectic, contact or volume forms $\alpha_{i}$. We show that if a group isomorphism between the automorphism groups of $\alpha_{i}$ is induced by a bijective map between $M_{i}$, then this map must be a $C^{\infty}$ diffeomorphism which exchanges the structures $\alpha_{i}$. This generalizes a theorem of Takens.
\end{abstract}

1. Introduction and statement of the main results. This paper is the first of a series devoted to showing that some classical geometric structures are determined by their automorphism groups: a leitmotiv of the Erlanger Program $[6]$.

Let $\left(M_{i}, \alpha_{i}\right), i=1,2$, be two smooth manifolds equipped with volume forms, symplectic forms or contact forms $\alpha_{i}$. Denote by $G_{\alpha_{i}}\left(M_{i}\right)$ the group of $C^{\infty}$ diffeomorphisms of $M_{i}$, which preserve $\alpha_{i}$ in case $\alpha_{i}$ are symplectic or volume forms, or which preserve $\alpha_{i}$ up to a function in case $\alpha_{i}$ are contact forms.

Our main result is

THEOREM 1. Let $\left(M_{i}, \alpha_{i}\right), i=1,2$, be two manifolds equipped with volume forms, symplectic forms or contact forms $\alpha_{i}$. Let $w: M_{1} \rightarrow M_{2}$ be a bijective map such that for any map $f: M_{1} \rightarrow M_{1}, w f w^{-1} \in G_{\alpha_{2}}\left(M_{2}\right)$ if and only if $f \in G_{\alpha_{1}}\left(M_{1}\right)$. Then $w$ is a $C^{\infty}$ diffeomorphism and $w^{*} \alpha_{2}=\lambda \alpha_{1}$ for some function $\lambda$, which is a constant if $\alpha_{i}$ are volume or symplectic forms.

This generalizes the following theorem of Takens [11].

THEOREM (TAKENS). Let $w: M_{1} \rightarrow M_{2}$ be a bijection between two smooth manifolds $M_{1}$ and $M_{2}$ such that $h: M_{1} \rightarrow M_{1}$ is a $C^{\infty}$ diffeomorphism iff whw $w^{-1}$ is a $C^{\infty}$ diffeomorphism. Then $w$ is a $C^{\infty}$ diffeomorphism.

We give a new and short proof of Takens' theorem. However our proof relies on a deep theorem of Montgomery-Zippin [8]. Then we show how the proof generalizes to the classical diffeomorphism groups mentioned in Theorem 1 to assert that $w$ must be a $C^{\infty}$ diffeomorphism. To show that this diffeomorphism exchanges the structures, we appeal to a theorem of Omori [9] generalizing the classical theorem of Pursell-Shanks [10], on Lie algebras of vector fields.

As a consequence of our main result and of a theorem of Wechsler [13], we obtain

THEOREM 2. Let $G_{\alpha_{i}}\left(M_{i}\right), i=1,2$, be as in Theorem 1. Suppose a group isomorphism $\Phi: G_{\alpha_{1}}\left(M_{1}\right) \rightarrow G_{\alpha_{2}}\left(M_{2}\right)$ is also a homeomorphism when $G_{\alpha_{i}}\left(M_{i}\right)$ are endowed with the point-open topology. Then there exists a $C^{\infty}$ diffeomorphism

Received by the editors September 13, 1985.

1980 Mathematics Subject Classification. Primary 53C15; Secondary 58D05, 57R50.

Key words and phrases. Automorphism of a geometric structure, Erlanger Program, symplectic forms, volume forms, contact structures, $\omega$-transitivity. 
$w: M_{1} \rightarrow M_{2}$ such that $\Phi(f)=w f w^{-1}, \forall f \in G_{\alpha_{1}}\left(M_{1}\right)$ and $w^{*} \alpha_{2}=\lambda \alpha_{1}$ for some function $\lambda$ which is a constant if $\alpha_{i}$ are volume or symplectic forms.

COROLLARY. If an automorphism of the group of contact diffeomorphisms $G_{\alpha}(M)$ of a contact manifold $(M, \alpha)$ is also a homeomorphism for the point-open topology, then it is an inner automorphism.

The goal of the second part of this paper [2] is to show that in the case $\alpha_{i}$ are volume forms and symplectic forms (under certain conditions), the hypothesis in our Theorem 2 that $\Phi$ is a homeomorphism for the point-open topology can be dropped. This uses an approach due to Filipkiewicz [5] and deep theorems on the structure of volume preserving and symplectic diffeomorphism groups $[\mathbf{1 2}, \mathbf{1}]$. We have not been so far in the contact case, the reason being that the structure of the group of contact diffeomorphisms is not well understood.

2. A new proof of Takens' theorem. One shows first that $w: M_{1} \rightarrow M_{2}$ must be homeomorphism. This is done exactly like in Takens [11] or Filipkiewicz [5]. For completeness we reproduce here the argument.

Let $\operatorname{Diff}^{\infty}(M)$ be the group of all $C^{\infty}$ diffeomorphisms of a smooth manifold $M$. Let $A$ denote the class of fixed subsets of elements of Diff ${ }^{\infty}(M)$, i.e. $A=$ $\left\{\operatorname{Fix}(f) \mid f \in \operatorname{Diff}^{\infty}(M)\right\}$ and $\operatorname{Fix}(f)=\{x \in M \mid f(x)=x\}$. Let $B$ be the class of complements of elements of $A$, i.e. $B=\{B=M-A, A \in A\}$. This is a class of open subsets of $M$. If $B \in B$, then $B$ is the interior of the support of some diffeomorphism. For any point $x$ belonging to an open set $U$ of $M$, it is easy to construct a diffeomorphism whose support contains $x$ and is contained in $U$, i.e. there exists $B \in B$ with $x \in B$ and $B \subset U$. This means that $B$ is a basis for the topology of $M$. If $h \in \operatorname{Diff}^{\infty}\left(M_{2}\right)$ then $\operatorname{Fix}\left(w h w^{-1}\right)=w(\operatorname{Fix}(h))$ and if $g \in \operatorname{Diff}{ }^{\infty}\left(M_{2}\right) \operatorname{Fix}\left(w^{-1} g w\right)=w^{-1}(\operatorname{Fix}(g))$. Hence $w$ and $w^{-1}$ take basic open sets into basic open sets: they are hence both continuous, i.e. $w$ is a homeomorphism.

We now want to show that $w$ and $w^{-1}$ are $C^{\infty}$ maps. Let $C^{\infty}(M)$ denote the set of $C^{\infty}$ real valued functions on a smooth manifold $M$. To prove that the continuous maps $w, w^{-1}$ are $C^{\infty}$, i.e. that $w$ is a $C^{\infty}$ diffeomorphism it is enough to show that $f \circ w \in C^{\infty}\left(M_{1}\right), \forall f \in C^{\infty}\left(M_{2}\right)$ and $g \circ w^{-1} \in C^{\infty}\left(M_{2}\right), \forall g \in C^{\infty}\left(M_{1}\right)[4]$. But the situation is symmetrical, so it is enough to show that $f \circ w \in C^{\infty}\left(M_{1}\right), \forall f \in$ $C^{\infty}\left(M_{2}\right)$.

The goal of the remainder of the proof is to provide explicit formulas for the partial derivatives of $f \circ w$.

Let $x \in M_{1}$ and $U$ be an open neighborhood of $x$ which is the domain of a local chart $\varphi: U \rightarrow \mathbf{R}^{n}$ (dimension of $M_{1}$ is $n$ ). The tangent bundle over $U,\left.T M\right|_{U}$, is trivial and let $\partial / \partial x_{i}, i=1, \ldots, n$, be the natural basis of any $T_{y} M_{1}, \forall y \in U$. Let $X_{i}, i=1,2, \ldots n$, be $C^{\infty}$ vector fields on $M_{1}$, with compact supports and which coincide with $\partial / \partial x_{i}$ on a neighborhood of $x$. To get $X_{i}$, simply multiply $\partial / \partial x_{i}$ with a $C^{\infty}$ function with compact support in $U$ and which is equal to 1 near $x$.

Let $h_{t}^{i}$ be the 1-parameter group of diffeomorphisms generated by $X_{i}$. Then $\bar{h}_{t}^{i}=\varphi h_{t}^{i} \varphi^{-1}: \varphi(U) \subseteq \mathbf{R}^{n} \rightarrow \varphi(U) \subseteq \mathbf{R}^{n}$ is given near $x$ by

$$
\bar{h}_{t}^{i}\left(x_{1}, x_{2}, \ldots, x_{n}\right)=\left(x_{1}, x_{2}, \ldots, x_{i-1}, x_{i}+t, x_{i+1}, \ldots, x_{n}\right) .
$$

Denote by $\overline{f \circ w}$ the local expression of $f \circ w$ in the chart $(\varphi, U)$, i.e. $\overline{f \circ w}=$ $f \circ w \circ \varphi^{-1}: \varphi(U) \rightarrow \mathbf{R}$. For $a \in U$ set $\varphi(a)=\left(a_{1}, a_{2}, \ldots, a_{n}\right)=\bar{a}$. Then, if $a \in U$ 
is near $x$ :

$$
\begin{aligned}
\partial / \partial x_{i}(\overline{f \circ w})(\bar{a}) & =\lim _{t \rightarrow 0} \frac{\left(f \circ w \circ \varphi^{-1}\right)\left(\bar{h}_{t}^{i}(\bar{a})\right)-\left(f \circ w \circ \varphi^{-1}\right)(\bar{a})}{t} \\
& =\lim _{t \rightarrow 0} \frac{(f \circ w)\left(h_{t}^{i}(a)\right)-(f \circ w)(a)}{t} \\
& =\left.\frac{d}{d t}(f \circ w)\left(h_{t}^{i}(a)\right)\right|_{t=0} .
\end{aligned}
$$

Let now $X$ be any $C^{\infty}$ vector field on $M_{1}$ with compact support and let $h_{t}$ be the 1-parameter group of diffeomorphisms generated by $X$. For each $t, H_{t}=w h_{t} w^{-1}$ is a $C^{\infty}$ diffeomorphism by hypothesis, and, the evaluation map:

$$
\mathscr{H}: \mathbf{R} \times M_{2} \rightarrow M_{2}:(t, x) \rightarrow H_{t}(x)=w h_{t} w^{-1}(x)
$$

is continuous. Moreover $H_{0}=$ identity and $H_{t+s}=H_{t} \circ H_{s}$. Therefore $\leftrightarrow: \mathbf{R} \times M_{2} \rightarrow$ $M_{2}(t, x) \rightarrow H_{t}(x)$ is a continuous action of $\mathbf{R}$ on $M_{2}$ by $C^{\infty}$ diffeomorphisms. By Theorem 3, $§ 5.2$ of Montgomery-Zippin [8, p. 212], since $\mathbf{R}$ is a Lie group, this action is $C^{\infty}$, i.e. $\forall t$ is smooth in both variables $t$ and $x$.

Therefore, the 1-parameter group of diffeomorphisms $H_{t}$ has an infinitesimal generator: i.e. a $C^{\infty}$ vector field $X_{w}$ with compact support such that $d H_{t}(x) / d t=$ $X_{w}\left(H_{t}(x)\right)$. Given $f \in C^{\infty}\left(M_{2}\right)$, its directional derivative $X_{w} \cdot f$ is a $C^{\infty}$ function. (Interpret $X_{w}$ as a derivation of the algebra $C^{\infty}\left(M_{2}\right)$.)

For any $x \in M_{1}$, we have

$$
\begin{aligned}
\left(X_{w} \cdot f\right)(w(x)) & =\left.\frac{d}{d t} f\left(H_{t}(w(x))\right)\right|_{t=0} \\
& =\left.\frac{d}{d t}(f \circ w)\left(h_{t}(x)\right)\right|_{t=0} .
\end{aligned}
$$

Therefore if $X$ is any of the vector fields $X_{i}$ above, we get, $\forall a \in U$ :

$$
\begin{aligned}
\frac{\partial}{\partial x_{i}}(\overline{(f \circ w)})(\bar{a}) & =\left.\frac{d}{d t}(f \circ w)\left(h_{t}^{i}(a)\right)\right|_{t=0} \\
& =\left(\left(X_{i}\right)_{w} \cdot f\right)(w(a)) .
\end{aligned}
$$

This formula shows that $f \circ w$ is a $C^{1}$ map, and that for any vector fields $X$ on $M_{1}$ with compact support, we have

$$
\left(X_{w} \cdot f\right) \circ w=X \cdot(f \circ w) .
$$

To compute higher partial derivatives, just iterate this formula using vector fields $X_{i}$. For instance

$$
\begin{aligned}
\left\{\left(X_{j}\right)_{w} \cdot\left[\left(X_{i}\right)_{w} \cdot f\right]\right\} \circ w & =X_{j} \cdot\left[\left(\left(X_{i}\right)_{w} \circ f\right) \circ w\right] \\
& =X_{j} \cdot\left(X_{i} \cdot(f \circ w)\right) .
\end{aligned}
$$

Therefore we have proved that $f \circ w \in C^{\infty}\left(M_{1}\right)$. This completes the proof of Takens' theorem.

REMARK. Starting with a $C^{\infty}$ vector field with compact support $X$ on $M_{1}$ and if $h_{t}$ is its 1-parameter group of diffeomorphisms, we have seen that thanks to Montgomery-Zippin, $H_{t}=w h_{t} w^{-1}$ is a 1-parameter group of diffeomorphisms, which is generated by $X_{w}$. Since $w$ is a $C^{\infty}$ diffeomorphism, we must have $X_{w}=$ $w_{*} X$ i.e. $\left(w_{*} X\right)(y)=\left(T_{w^{-1}(y)} w\right)\left(X\left(w^{-1}(y)\right)\right), \forall y \in M_{2}$. Thus $w$ induces a Lie algebra isomorphism between the Lie algebras of compactly supported vector fields on $M_{1}$ and $M_{2}$ by $X \rightarrow X_{w}$. 
3. Generalizations: Proof of Theorem 1. For any point $x$ belonging to an open set $U$ of $M_{i}$ it is easy to construct an $h \in G_{\alpha_{i}}\left(M_{i}\right)$ such that $x \in \operatorname{Int}(\operatorname{supp}(h))$ and $\operatorname{supp}(h) \subset U$. Therefore, the same argument as in the proof of Takens' theorem shows that $w$ must be a homeomorphism.

Let $\mathcal{L}_{\alpha_{i}}\left(M_{i}\right)$ be the Lie algebra of vector fields with compact supports on $M_{i}$, generating 1-parameter groups of diffeomorphisms $h_{t}$ belonging to $G_{\alpha_{i}}\left(M_{i}\right)$. For each $X \in \mathcal{L}_{\alpha_{1}}\left(M_{1}\right)$ and $f \in C^{\infty}\left(M_{2}\right)$, we have

$$
\left.\frac{d}{d t}(f \circ w)\left(h_{t}(x)\right)\right|_{t=0}=\left(X_{w} \cdot f\right)(w(x))
$$

where $X_{w}$ is defined as in the proof of Takens' theorem and $h_{t}$ is the 1-parameter group of diffeomorphisms generated by $X$.

As before, we want to use $(*)$ to compute partial derivatives of $f \circ w$.

We consider first the volume preserving and symplectic cases. Let $x \in M_{1}$ and $U$ a contractible open neighborhood of $x$ which is the domain of a local canonical $\operatorname{chart} \varphi: U \rightarrow \mathbf{R}^{n}\left(n=\operatorname{dim} M_{1}\right):$ in this chart, $\left.\alpha_{1}\right|_{U}=\varphi^{*}(\underline{\alpha})$ where

$$
\underline{\alpha}=d x_{1} \wedge d x_{2}+d x_{3} \wedge d x_{4}+\cdots+d x_{2 m-1} \wedge d x_{2 m} \quad(n=2 m)
$$

in the symplectic case and

$$
\underline{\alpha}=d x_{1} \wedge d x_{2} \wedge d x_{3} \wedge \cdots \wedge d x_{n}
$$

in the volume case. The existence of these canonical charts are guaranteed by Darboux's theorem.

The vector fields $\left(\partial / \partial x_{k}\right)$ defined on $U$ can be extended into elements of $\mathcal{L}_{\alpha_{1}}\left(M_{1}\right)$ [9]. Indeed, clearly $L_{\left(\partial / \partial x_{k}\right)} \alpha_{1}=0$, when $L_{y} \alpha$ is the Lie derivative of $\alpha$ in the direction of a vector field $y$. Hence $d \mathrm{i}\left(\partial / \partial x_{k}\right) \alpha_{1}=0$, here $\mathrm{i}(X) \alpha$ is the interior product of $\alpha$ and $X$. By Poincaré's lemma, there exists a function (symplectic case) or an $(n-2)$-form (volume case) $B_{k}$ with: $\mathrm{i}\left(\partial / \partial x_{k}\right) \alpha_{1}=d B_{k}$. Let $\lambda$ be a smooth function with compact support in $U$ and $\lambda=1$ near $x$. The vector fields $X_{k}$ extending $\partial / \partial x_{k}$ may then be defined by

$$
\mathrm{i}\left(X_{k}\right) \alpha_{1}=d\left(\lambda B_{k}\right) \text {. }
$$

Therefore in these cases we can use $(*)$ to get all the partial derivatives of $f \circ w$.

In the contact case, the canonical chart around $x, \varphi: U \rightarrow \mathbf{R}^{n}(n=2 m+1)$ is such that $\left.\alpha_{1}\right|_{U}$ is a multiple of $\varphi^{*} \underline{\alpha}$ with

$$
\underline{\alpha}=d z-\left(y_{1} d x_{1}+y_{2} d x_{2}+\cdots+y_{m} d x_{m}\right) .
$$

On $U$ consider the vector fields given by

$$
Z=\partial / \partial z, \quad X_{k}=\partial / \partial x_{k}, \quad Y_{k}=\partial / \partial y_{k}+x_{k} \partial / \partial z
$$

Clearly, if $\xi$ is one of the vector fields above, $L_{\xi} \underline{\alpha}=0$. We now want to show that these vector fields defined on $U$ can be extended into elements of $\mathcal{L}_{\alpha_{1}}\left(M_{1}\right)$ [9].

It is well known that a contact vector field $\xi$ on a contact manifold $(M, \alpha)$ is completely determined by the function $\mathrm{i}(\xi) \alpha[7]$. Therefore if $\lambda$ is a $C^{\infty}$ function which is equal to 1 near $x$ and has compact support in $U$, the function $\lambda(\mathrm{i}(\xi) \alpha)$ (where $\xi$ is one of the vector fields above) determines contact vector fields $\bar{Z}, \bar{X}_{k}, \bar{Y}_{k}$ which have compact supports and coincide with $Z, X_{k}, Y_{k}$ near $x$. As in the proof 
of Takens' theorem, we can compute the partial derivatives of $\overline{f \circ w}$, the local expansion of $f \circ w$ within the canonical charts as follows:

$$
\begin{aligned}
\partial / \partial z(\overline{f \circ w}) & =\left(\bar{Z}_{w} \cdot f\right) w(x) \\
\partial / \partial x_{k}(\overline{f \circ w}) & =\left(\left(\bar{X}_{k}\right)_{w} \cdot f\right) w(x) \\
\partial / \partial y_{k}(\overline{f \circ w}) & =\left(\left(\bar{Y}_{k}\right)_{w} \cdot f\right) w(x)-x_{k}\left(\left(\bar{Z}_{w}\right) \cdot f\right) w(x) .
\end{aligned}
$$

To compute higher derivatives, apply again these formulas with $f$ replaced by $\bar{Z}_{w} \cdot f,\left(\bar{X}_{k}\right)_{w} \cdot f,\left(\bar{Y}_{k}\right)_{w} \cdot f$, etc. We thus have proved that $f \circ w$ is $C^{\infty}$ and hence $w$ is a $C^{\infty}$ diffeomorphism.

The last task is now to show that $w$ exchanges the structures.

As we have already observed, $\forall X \in \mathcal{L}_{\alpha_{1}}\left(M_{1}\right)$ we get $X_{w} \in \mathcal{L}_{\alpha_{2}}\left(M_{2}\right)$ such that $X_{w}=w_{*} X$. Therefore $w$ induces a Lie algebra isomorphism between the Lie algebras $\mathcal{L}_{\alpha_{i}}\left(M_{i}\right)$.

By a theorem of Omori [9], generalizing a classical result of Pursell-Shanks [10], there exists a $C^{\infty}$ diffeormorphism $\rho: M_{1} \rightarrow M_{2}$ inducing the isomorphism $X \rightarrow$ $X_{w}=w_{*} X$ and such that $\rho^{*} \alpha_{2}=\lambda \alpha_{1}$ for some function $\lambda$ which is a constant if $\alpha_{i}$ are symplectic or volume forms. But the condition $\rho_{*} \xi=w_{*} \xi, \forall \xi \in \mathcal{L}_{\alpha_{1}}\left(M_{1}\right)$ implies that $\rho=w$. Indeed if $\varphi=\rho^{-1} w$ and $h_{t}$ is the 1-parameter group of diffeomorphisms generated by $\xi$, then $\varphi h_{t} \varphi^{-1}=h_{t}$. But this implies that $\varphi=$ identity, i.e. $\rho=w$. Indeed if $\varphi \neq$ identity, let $x \in M_{1}$ with $\varphi(x)=y \neq x$. Choose $\xi \in \mathcal{L}_{\alpha_{1}}\left(M_{1}\right)$ with $\xi(x) \neq 0$, with a support not containing $y$. If $h$ is the time-one flow of $\xi$, then: $h(x)=z \neq x($ since $\xi(x) \neq 0)$ and $\varphi h \varphi^{-1}(y)=\varphi h(x)=$ $\varphi(z)$. But $h(y)=y=\varphi(x) \neq \varphi(z)$ since $x \neq z$, i.e. $\varphi h \varphi^{-1} \neq h$. Contradiction. The proof of Theorem 1 is now complete.

4. Applications. A group of homeomorphisms $G(X)$ of a topological space $X$ is said to be $\omega$-transitive on $X$ if for each $n \in \mathbf{N}$ and each pair on $n$-tuples of distinct points $\left(x_{1}, x_{2}, \cdots, x_{n}\right),\left(y_{1}, y_{2}, \cdots, y_{n}\right)$, there exists a homeomorphism $h \in G(X)$ such that $h\left(x_{i}\right)=y_{i}$.

In [13], Wechsler has proved a theorem which contains the following result:

THEOREM (WECHSLER). Let $\phi: G\left(M_{1}\right) \rightarrow G\left(M_{2}\right)$ be a group isomorphism between two groups of diffeomorphisms of two smooth connected manifolds $M_{i}$, of dimension $n \geq 2$. Suppose that $G\left(M_{i}\right)$ are $\omega$-transitive on $M_{i}$ and that $\phi$ is a homeomorphism when $G\left(M_{i}\right)$ are endowed with the point-open topology. Then, there exists a homeomorphism $w: M_{1} \rightarrow M_{2}$ such that $\phi(f)=w \circ f \circ w^{-1}, \forall f \in G\left(M_{1}\right)$.

ProOF OF THEOREM 2. By Boothby's theorem [3], the groups considered in Theorem 2 are $\omega$-transitive. Therefore by Wechsler's theorem, there exists a homeomorphism $w: M_{1} \rightarrow M_{2}$ such that $\varphi(f)=w f w^{-1}, \forall f \in G_{\alpha_{1}}\left(M_{1}\right)$. By our Theorem $1, w$ is a $C^{\infty}$ diffeomorphism and exchanges the structures $\alpha_{i}$.

\section{REFERENCES}

1. A. Banyaga, Sur la structure du groupe des diffeomorphismes qui preservent une forme symplectique, Comment. Math. Helv. 53 (1978), 174-227.

2. $\ldots$, On isomorphic classical diffeomorphism groups. II, preprint, 1985.

3. W. M. Boothby, Transitivity of automorphisms of certain geometric structures, Trans. Amer. Math. Soc. 137 (1969), 93-100. 
4. Th. Brocker and K. Janich, Introduction to differential topology, Cambridge Univ. Press, New York, 1982.

5. R. P. Filipkiewicz, Isomorphisms between diffeomorphism groups, Ergodic Theory Dynamical Systems 2 (1982), 159-171.

6. F. Klein, Erlanger programm, Math. Ann. 43 (1893), 63.

7. V. V. Lychagin, Local classification of non linear first order partial differential equations, Russian Math. Surveys 30 (1975), 105-175.

8. D. Montgomery and L. Zippin, Topological transformation groups, Wiley, Chichester, 1955.

9. H. Omori, Infinite dimensional Lie transformation groups, Lecture Notes in Math, vol. 427, Springer-Verlag, Berlin and New York, 1974.

10. L. E. Pursell and M. E. Shanks, The Lie algebra of smooth manifolds, Proc. Amer. Math. Soc. (1954), 468-472.

11. F. Takens, Characterization of a differentiable structure by its group of diffeomorphisms, Bol. Soc. Brasil. Mat. 10 (1979), 17-25. MR 82e-58027.

12. W. Thurston, On the structure of volume preserving diffeomorphisms, unpublished.

13. Mr. Wechsler, Homeomorphism groups of certain topological spaces, Ann. of Math. 2 (1955), 360-373.

Department of Mathematics, The Pennsylvania State University, UniverSITY PARK, PENNSYLVANNIA 16802 